\title{
Acute Kidney Injury in Pregnancy
}

\author{
Manisha Sahay \\ Department of Nephrology \\ Osmania Medical College and General Hospital, Hyderabad, Andhra Pradesh \\ India
}

\section{Introduction}

Acute kidney injury (AKI) is a life threatening complication of pregnancy.The incidence of AKI has sharply declined from 0.5 per 1000 pregnancies to one in 20,000 births in developed countries. (Beaufilis,2005) On the other hand, pregnancy is still responsible for $15-20 \%$ of AKI in developing countries. (Naqvi, 1996)Pregnancy related AKI (PRAKI) is on the decline from $14.5 \%$ reported in 1987 to $4.3 \%$ in 2005 in India(Chugh,1987).

\subsection{Physiological changes in pregnancy}

\subsubsection{Renal function during pregnancy}

The kidney undergoes monumental physiologic and anatomic changes during a normal pregnancy. Renal plasma flow increases by $50-70 \%$. Plasma volume increases by $50 \%$ and there is hemodilutional anemia. Cardiac output increases by $40 \%$.Glomerular Filtration Rate (GFR) is maximum around the 13th week of pregnancy and can reach levels up to $150 \%$ of normal. Despite increased GFR the intraglomerular pressure remains normal. Serum creatinine falls by an average of $0.4 \mathrm{mg} / \mathrm{dl}$ to a pregnancy range of 0.4 to 0.8 $\mathrm{mg} / \mathrm{dl}$. Hence, a serum creatinine of $1.0 \mathrm{mg} / \mathrm{dl}$, although normal in a non pregnant individual, reflects renal impairment in a pregnant woman. Serum creatinine rises near term and value of $1 \mathrm{mg} / \mathrm{dl}$ is considered normal. In the initial part of pregnancy there is decreased peripheral vascular resistance with a blood pressure fall of approximately 10 $\mathrm{mm} \mathrm{Hg}$ in the first 24 weeks. The blood pressure gradually returns to prepregnancy level by term. Glycosuria occurs due to decrease in transport maximum for glucose(TMG) and high GFR. Aminoaciduria $(2 \mathrm{~g} / \mathrm{d})$ may be seen. Increased uric acid clearance results in low uric acid level $(2.5-5.5 \mathrm{mg} / \mathrm{dl})$ but levels increase later and reach prepregnancy values at term. A value of $>6 \mathrm{mg} / \mathrm{dl}$ reflects pregnancy induced hypertension (PIH). Potassium and almost $900 \mathrm{meq}$ of sodium are retained. Calcium excretion increases but stone formation is not increased as there is increased excretion of inhibitors of stone formation. A reset in the osmostat occurs, resulting in increased thirst and decreased serum sodium levels ( by $5 \mathrm{mEq} / \mathrm{L}$ ) and low plasma osmolality (10 m0sm/ kg less ). Clearance of $\mathrm{ADH}$ is increased by placental vasopressinase and may result in transient Diabetes insipidus of pregnancy which may respond to DDAVP. On the other hand there are some reports of transient SIADH in pregnancy. Urine concentration and dilution are adequate. There is mild respiratory alkalosis and blood gas of 7.42-7.44/30 pCO2/HCO318-22 is representative (Chris Baylis,2007). 


\subsubsection{Anatomic changes}

A dilatation of ureters and pelvis occurs till pelvic brim (iliac sign) with dilatation more pronounced on right secondary to dextrorotation of uterus and dilatation of right ovarian venous plexus. This leads to urinary stasis and an risk of urinary tract infections (UTIs). There is an increase in kidney size by $1-1.5 \mathrm{~cm}$. As a rule, all the physiologic changes maximize by the end of the second trimester and then start to return to the prepartum level, whereas changes in the anatomy take up to 3 months postpartum to subside.(Hou, S . 1998, Chris Baylis, 1987)

\subsubsection{Hormones}

There is increase in aldosterone, desoxycorticosterone, progesterone, relaxin, oxytocin and vasodilating prostaglandins and a decrease in vasopressin (due to vasopressinase) and also resistance to action of aldosterone and renin.(August, P 1995)

\subsubsection{Renal function tests}

Urine examination- Microscopic hematuria may be seen in $20 \%$ but is not persistent and disappears after delivery. Proteinuria glycosuria, and hypercalciuia may be seen.

\section{Glomerular filtration rate (GFR) -}

MDRD formula- Creatinine-based formulae are inaccurate in pregnancy. The Modification of Diet in Renal Disease (MDRD) formula underestimates GFR by $40 \mathrm{ml} / \mathrm{min}$. Among pregnant women with preeclampsia or CKD, MDRD formula is slightly better, underestimating GFR by 23.3 and $27.3 \mathrm{ml} / \mathrm{min}$ respectively.

Creatinine clearance by24-h urine collection closely approximates GFR by inulin clearance among healthy pregnant women and is the gold standard in pregnancy.

Weight-based formulas such as Cockroft-Gault formulae overestimate GFR by approximately $40 \mathrm{ml} / \mathrm{min}$.

\section{Estimating of proteinuria during pregnancy}

Urine protein excretion increases in pregnancy and upto $300 \mathrm{mg} /$ day is normal. Albumin excretion is also increased. These values return to normal by $6^{\text {th }}$ month post partum. Twentyfour-hour urine collection, although the gold standard for proteinuria quantification is cumbersome, inaccurate and result is delayed. The use of the protein to creatinine ratio ( $\mathrm{P}: \mathrm{C}$ ratio) to estimate24-h protein excretion is controversial in pregnancy though it has become the preferred method for the quantification of proteinuria in non pregnant population, because of high accuracy, reproducibility, and convenience. Most misclassifications occur in women with borderline proteinuria ( 250 to $400 \mathrm{mg} / \mathrm{d}$ ). Hence, it is reasonable to use urine $\mathrm{P}: \mathrm{C}$ ratio for diagnosis, with 24-h collection undertaken when result is equivocal.

Renal biopsy in pregnancy - Indications include severe symptomatic nephrotic syndrome and rapidly progressive renal failure. Biopsy can be done in $2^{\text {nd }}$ trimester with patient in lateral position.

\section{Acute kidney injury in pregnancy}

Certain renal diseases are common in pregnancy. (Ananth Karumanchi,S 2007, Schrier RW 1997). Incidence of AKI in pregnancy is 1:20,000 and comprises $25 \%$ of AKI in developing countries with substantial mortality. Acute renal failure in pregnancy can be induced by any of the disorders leading to renal failure in the general population, such as acute tubular necrosis due to infection, glomerulonephritis related to lupus, or drug toxicity. There are, 
however, pregnancy complications characteristic of each trimester that can result in renal failure.

\subsection{Causes of renal failure in pregnancy}

Can be divided into

i. Early pregnancy- Hyperemesis gravidarum, Septic abortion .

ii. Late pregnancy-PIH and its complications, Hemolytic anemia ,elevated liver enzymes and low platelets (HELLP), Post partum Hemolytic uremic syndrome (HUS), Acute fatty liver of pregnancy, Volume loss -Antepartum hemorrhage (APH), Post partum hemorrhage (PPH), Sepsis

In a study carried out at Osmania General hospital ,Hyderabad ,India over a period of 8 years Obstetric renal failure accounted for $12.1 \%$ with PIH as the commonest cause accounting for $39.3 \%$, PPH 20.7\% ,Puerperal sepsis 10.6\%, APH-9.2\%.Septic abortion$4.9 \%$, HUS- $3.4 \%$ and $11.7 \%$ were undetermined .

\section{Early pregnancy causes}

\subsubsection{Hyperemesis gravidarum}

Severe vomiting in early pregnancy can lead to volume depletion and acute kidney injury. Metabolic alkalosis can be seen. Vomiting can be aggravated by certain triggers which include strong smells, postures which delay gastric emptying, hot foods etc. The patient may need hospitalization and volume replacement. Antiemetic drugs can be used to control vomiting. These include pyridoxine-doxylamine succinate combination therapy for initial pharmacologic treatment of nausea of pregnancy, antihistaminics, domperidone, metaclopramide, and ondensetron. Glucocorticoids have been used in refractory cases. Hyperemesis may recur in subsequent pregnancies. (ACOG, 2004)

\subsubsection{Septic abortion}

The commonest causative organism is Clostridium. It manifests few hours to 1-2 days after abortion with fever, vomiting and pain abdomen. Progression to shock and death is rapid. Jaundice due to hemolysis and cutaneous vasodilatation contribute to bronze skin coloration. Anemia, leucocytosis and thrombocytopenia with associated disseminated intravascular coagulation may be seen. Management consists of antibiotics and volume resuscitation. Other therapies include hysterectomy, hyperbaric oxygen, antitoxin and exchange transfusion.

\section{Late pregnancy causes}

\subsubsection{Hypertensive disorders of pregnancy}

Hypertensive disorders are the commonest cause of renal failure in pregnancy. These are seen in two settings: young primigravidas and older multiparous women.

\subsubsection{Classification}

Terminology of hypertensive disorders varies, but following 5 entities have been described by National High Blood Pressure Education Program (NHBPEP, 2000):

Gestational hypertension/ Transient hypertension - is defined as blood pressure of 140/90 $\mathrm{mm} \mathrm{Hg}$ or greater with no hypertension before pregnancy. It usually affects nulliparous females mostly in third trimester. Preeclampsia does not develop and blood pressure returns to normal levels within 12 weeks postpartum. Patients are usually asymptomatic or 
have symptoms or signs like preeclampsia. It is a retrospective diagnosis and is confused with preeclampsia at the time of onset and with essential hypertension postpartum until the blood pressure returns to normal. Proteinuria does not occur, serum uric acid is normal. It may predict the development of hypertension later in life. It should be managed as preeclampsia when first diagnosed.

Chronic hypertension- is associated with underlying or preexisting hypertension. The diagnosis is established by a blood pressure of $140 / 90 \mathrm{~mm} \mathrm{Hg}$ or greater before pregnancy or before 20 weeks' gestation, or by persistent hypertension after delivery. Renal biopsy shows nephrosclerosis rather than preeclampsia. Complications of chronic hypertension include superimposed preeclampsia and renal failure, abruptio placentae, growth restriction and fetal death. Methyldopa is the preferred treatment agent, but in mild cases, no therapy may be necessary. In women whose blood pressure is well controlled when they enter pregnancy, the same therapy should be continued. However, angiotensin II receptor blocking (ARB) agents and angiotensin converting enzyme inhibitors (ACEIs) are contraindicated in pregnancy due to the risk of fetal renal agenesis.

Preeclampsia - It is the leading cause of acute kidney injury in the last trimester and occurs in $7 \%$ of all pregnancies mostly in primigravidas. However it can be seen in multigravidas if the interpregnancy interval is prolonged. It is the leading cause of maternal and fetal mortality in the world. It is associated with intrauterine growth retardation and small for gestational age (SGA) babies. It is a triad of hypertension, proteinuria and oedema occurring after the $20^{\text {th }}$ week of gestation with few cases developing postpartum within hours, usually in the first 24 to 48 hours. Preeclampsia does not occur before twenty weeks gestation except in molar pregnancies and in the presence of antiphospholipid antibody syndrome. Hypertension is defined as rise in systolic $\mathrm{BP}>30 \mathrm{mmHg}$ and diastolic $\mathrm{BP}>15$ $\mathrm{mmHg}$. Proteinuria is defined as $>300 \mathrm{mg}$ protein in urine per day. Oedema is not essential for diagnosis but sudden oedema and weight gain are common presenting features. Oedema is due to overfill with reduced GFR as in nephritic illnesses with suppressed renin and aldosterone and differs from oedema of $\mathrm{CHF}$, cirrhosis and neprhotic syndrome which is associated with underfilling of vascular bed with high renin and aldosterone. Though increased capillary permeability and hypoalbuminemia may be seen they are not the sole contributing factors for oedema. The risk factors associated with the development of preeclampsia include age older than 35 years or younger than 16 years, chronic hypertension, obesity, preexisting diabetes, renal disease, insulin resistance, Anti phospholipid antibody syndrome, African American race and pregnancy with a new partner. Fetal risk factors may be twin or multiple gestations, trisomy 13 and hydatidiform mole. Some genetic factors contribute to the development of preecclampsia. It is four times more common if there is history of precclampsia in mother or sister. Polymorphisms of genes associated with control of blood pressure or coagulation system have been implicated. Some mutations described involve Endothelial nitric oxide synthetase (e NOS), renin angiotensinogen (T235), Leyden factor V, Prothrombin and Methyl tetra hydrofolate reductase(MTHFR) genes. A locus on $2 p$ was described in Icelandic and Australian families. A Dutch study reported linkage to $12 \mathrm{q}$ locus in HELLP syndrome which shows the genetic factors responsible for Preclampsia and HELLP may be different. Association with trisomy 13 indicates a linkage to chromosome 13. An activating mutation of mineralocorticoid receptor was described in patients with PIH but no proteinuria. Preclampsia is more commonly seen in women at high altitude or in third world countries suggesting the possible role of hypoxia or environmental pollution in genesis. HELLP syndrome 
(Hemolysis, ELevated liver enzymes, and Low Platelets) is observed when severe preeclampsia or eclampsia is accompanied by significant liver involvement. Acute renal failure (ARF) may develop.(Lindheimer, 1991)

Eclampsia-is the occurrence of seizure activity with no other explainable cause in setting of preeclampsia.

Preeclampsia superimposed on chronic hypertension- defined as new-onset proteinuria (ie, $>300 \mathrm{mg} / \mathrm{d}$ ) after 20 weeks' gestation in a hypertensive patient or as a sudden increase in proteinuria or blood pressure in a patient with hypertension and proteinuria before 20 weeks' gestation.

\subsubsection{Pathophysiology}

Utero-placental ischemia: A combination of genetic and environmental factors results in inadequate invasion of uterine spiral arteries by placental trophoblasts resulting in inability of uterine vessels to transform from high resistance channels to a low resistance system. This results in utero-placental ischemia. In normal pregnancy Vascular endothelial growth factor (VEGF) and Placental induced growth factor (PIGF) are made by placenta and circulate in high concentrations in the blood. VEGF is also synthesized by glomerular podocytes and vascular endothelial cells. VEGF and PIGF induce synthesis of nitric oxide and vasodilating prostacylin in endothelial cells decreasing vascular tone and blood pressure. In PIH the utero-placental ischemia results in oxidative stress and release of a soluble cytokine, the sFlt-1 (soluble fms-like tyrosine kinase-1). sFlt-1 is a potent antagonist of VEGF and PIGF. In PET concentrations of sFlt-1 rise in the 2nd trimester with a decrease in VEGF and PIGF. Overproduction of sFlt-1 explains increased susceptibility to PET in multiple gestation, hydantidiform mole, trisomy 13 and first pregnancy. In addition, increased concentration of circulating pre-ecclamptic factorsangiotensin 1 / Bradykinin B2 receptor heterodimers, agonistic antibodies to angiotensin I receptor(AT 1 receptor antibody) and soluble endoglin are seen in PET. (Maynard,S 2003; Venkatesha,S 2006; Zhou,C.C,2008; Levine, RJ 2006)

There is an imbalance of vasoconstrictors and vasodilator substances hence there is a state of generalized vasoconstriction in preeclampsia. The levels of prorenin, angiotensin, thromboxane and endothelin are high while the levels of vasodilator prostaglandin ie Prostacyclin, PGE2, nitric oxide and bradykinin are low. In normal pregnancy there is less sensitivity of vascular bed to the hypertensive effects of angiotensin but this is lost in preeclampsia.

A myriad of markers for generalized endothelial dysfunction are seen in preeclampsia which include von Willebrand factor, Platelet activating factor (PAF-1), Endothelin and cellular fibronectin.

The increase in blood volume, increased GFR and vasodilatation seen in normal pregnancy are lost in preeclampsia. Though the blood volume in preeclampsia is decreased there is an increase in effective circulating volume with suppressed renin and aldosterone with elevation of brain natriuretic peptide.

\subsubsection{Screening tests}

Laboratory evaluation helps to determine disease severity by characterizing the extent of end organ involvement.

Hematocrit - Hemoconcentration supports the diagnosis of preeclampsia but hemolysis, if present, can decrease the hematocrit. 
Platelet count - Preeclampsia accounts for $21 \%$ of cases of maternal thrombocytopenia. Thrombocytopenia is usually moderate and platelet count rarely decreases to $<20,000 / \mu \mathrm{L}$. Thrombocytopenia in patients with preeclampsia always correlates with the severity of the disease. It is considered a sign of worsening disease and is an indication for delivery.

Quantification of protein excretion - Excretion of $300 \mathrm{mg}$ or more in 24 hours is necessary for diagnosis. It is suggested by at least $1+$ protein on dipstick of two urine specimens collected at least four hours apart. A dipstick of $3+$ or greater or $5 \mathrm{~g}$ or more per day is a criterion of severe disease.

Serum creatinine concentration - An elevated or rising level suggests severe disease.

Serum alanine and aspartate aminotransferase concentrations (ALT and AST) - Elevated or rising levels suggest hepatic dysfunction indicative of severe disease.

Serum lactate dehydrogenase (LDH) concentration - Microangiopathic hemolysis is suggested by an elevated LDH level and red cell fragmentation (schistocytes or helmet cells) on peripheral blood smear. Elevation of total bilirubin may also suggest hemolysis. Microangiopathic hemolysis is present in severe disease or HELLP syndrome (Hemolysis, Elevated Liver function tests, Low Platelets).

Serum uric acid concentration - It is often elevated in preeclampsia, but not diagnostic. High uric acid levels may be due to hypoxia or due to reduced GFR.

Fetal well-being is evaluated by a nonstress test or biophysical profile. In addition, the fetus is examined by ultrasound to evaluate growth and amniotic fluid volume. Assessment of umbilical artery Doppler flow may also aid in evaluation of the fetal condition.

Coagulation function tests (eg, prothrombin time, activated partial thromboplastin time, fibrinogen concentration) are usually normal if there is no thrombocytopenia or liver dysfunction, and therefore do not need to be monitored routinely.

Recently, urinary PIGF values and sFlt-1 values have shown great promise as a means of identifying preclinical preeclampsia .

\subsubsection{Management}

The definitive treatment of preeclampsia is delivery to prevent development of maternal or fetal complications from disease progression. Several groups have formulated guidelines for diagnosis, evaluation, and management of hypertensive disorders of pregnancy (NHBPEP. Report of the National High Blood Pressure Education Program Working Group on High Blood Pressure in Pregnancy (2000), ACOG 2002).

Laboratory follow-up - The minimum laboratory evaluation should include platelet count, serum creatinine, and serum AST. These tests should be repeated once or twice weekly in women with mild preeclampsia to assess for disease progression, and more often if clinical signs and symptoms suggest worsening disease(ACOG 2000). The value of other tests is less clearly defined. A rising hematocrit can be useful to look for hemoconcentration, which suggests contraction of intravascular volume and progression to more severe disease, while a falling hematocrit may be a sign of hemolysis. An elevated serum LDH concentration is also a sign of hemolysis, and a marker of severe disease or HELLP syndrome. Hemolysis can be confirmed by observation of schistocytes and helmet cells on a blood smear .Quantification of protein excretion can be performed to determine whether the threshold for severe preeclampsia ( $5 \mathrm{~g} / 24$ hours) has been reached. Since several clinical studies have shown that neither the rate of increase nor the amount of proteinuria affects maternal or perinatal outcome in the setting of preeclampsia, repeated 24-hour urinary protein estimations are not useful once the threshold of $300 \mathrm{mg} / 24$ hours for the diagnosis of 
preeclampsia has been exceeded. Serum creatinine alone can be used to monitor renal function.

Treatment of hypertension - Nonpharmacologic treatments include bed rest and alcohol avoidance. Weight loss and salt restriction are not recommended. ACE inhibitors are contraindicated. Diuretics in pregnancy lead to intravascular volume depletion, organ and placental hypoperfusion. They may be used with caution in presence of significant edema. If preeclampsia develops diuretics must be discontinued. Beta-blockers have no major contraindications, although neonatal bradycardia, hypoglycemia, and respiratory depression can occur. Labetalol is not associated with neonatal bradycardia and is used for hypertensive emergencies. Alpha-methyldopa is the drug of choice for hypertension. Clonidine is also used. Calcium channel blockers may cause tocolysis in $3^{\text {rd }}$ trimester hence should be used only if hypertension is unresponsive to other medications. Hydralazine is a first-line agent for hypertensive emergencies. Experience with minoxidil and prazosin is limited. The use of antihypertensive drugs to control mildly elevated blood pressure in the setting of preeclampsia does not alter the course of the disease or diminish perinatal morbidity or mortality. Novel therapies are under investigation. L-arginine is the physiologic precursor for nitric oxide, which has been implicated in the pathogenesis of preeclampsia and is being tested. Another randomized trial showed that plasma volume expansion did not improve maternal or fetal outcome.

Assessment of fetal well-being - A minimum of daily fetal movement counts and twice weekly fetal non stress testing with assessment of amniotic fluid volume, or biophysical profile is recommended. Early fetal growth restriction may be the first manifestation of preeclampsia or a sign of severe preeclampsia. Sonographic estimation of fetal weight should be performed to look for growth restriction and oligohydramnios at the time of diagnosis of preeclampsia and then repeated serially. Doppler velocimetry is useful for assessing fetal status if fetal growth restriction is present.

Delivery- The decision to deliver the fetus is based upon gestational age, maternal and fetal condition, and the severity of preeclampsia.

Mild PIH- Patients at term ( $>37$ weeks) are delivered. Most experts advise that delivery should not be postponed beyond 40 weeks of gestation in any preeclamptic woman. Vaginal delivery is preferred at term if there are no contraindications. Cervical ripening agents should be considered in women with unfavorable cervices. Women with mild PIH with period of gestation $<37$ weeks can be managed expectantly. However maternal end-organ dysfunction and non reassuring tests of fetal well-being may be indications for delivery at any gestational age. Outpatient care is a cost-effective option for some women with mild preeclampsia. However these patients should comply with frequent maternal and fetal evaluations (every one to three days) and have ready access to medical care. Restricted activity is recommended; however, complete bed rest is not required. Hospitalization is needed in the event of fulminant progression to eclampsia, hypertensive crisis, abruptio placentae, or HELLP syndrome. Patients should be hospitalized immediately if they develop severe or persistent headache, visual changes, right upper quadrant or epigastric pain, nausea or vomiting, shortness of breath, or decreased urine output (ACOG 2000). As with any pregnancy, decreased fetal movement, vaginal bleeding, abdominal pain, rupture of membranes, or uterine contractions should be reported immediately.

Severe preeclampsia - Severe preeclampsia is generally regarded as an indication for delivery, regardless of gestational age, to minimize the risk of development of maternal and fetal complications. Prolonged antepartum management at a tertiary care setting or in 
consultation with a maternal-fetal medicine specialist may be considered in few women under 32 to 34 weeks of gestation. However, women who develop severe preeclampsia at or beyond 32 to 34 weeks of gestation should be delivered at an institution with appropriate facilities. Hyaline membrane disease is common in preterm infants of preeclamptic women. Therefore, antenatal corticosteroids (betamethasone) to promote fetal lung maturity should be administered to women less than 34 weeks of gestation since preterm delivery is common. Even in preterm delivery vaginal delivery may be attempted. Cervical ripening agents may be used prior to induction if the cervix is not favorable. However, a prolonged induction should be avoided. Some authors recommend cesarean delivery for women with severe preeclampsia who are under 30 weeks of gestation. Management of severe preeclampsia- or eclampsia-related pulmonary edema includes treatment of the severe preeclampsia or eclampsia, supplemental oxygen, and fluid restriction. Diuresis is indicated if there is fluid overload but care should be taken to avoid further intravascular volume depletion. Mechanical ventilation may be necessary. Close, continuous maternal-fetal monitoring is indicated intrapartum to identify worsening hypertension, deteriorating maternal hepatic, renal, cardiopulmonary, or hematologic function, and uteroplacental insufficiency or abruptio placentae (often manifested by fetal bradycardia/or vaginal bleeding). The vitals should be monitored every hour and preeclampsia laboratory tests should be repeated every six hours. Epidural anaesthesia should be avoided in presence of thrombocytopenia. Invasive hemodynamic monitoring can be useful in complicated patients. Anticonvulsant therapy is generally initiated during labor or while administering corticosteroids or prostaglandins prior to planned delivery. Therapy is continued for 24 hours postpartum (range 12 to 48 hours). Magnesium sulfate is the drug of choice for the prevention of eclampsia and prevention of recurrent eclamptic seizures. It is more effective than Phenytoin or Nimodipine or lytic cocktail ( mixture of chlorpromazine, promethazine and pethidine). Magnesium causes vasodilatation of the cerebral vasculature, inhibition of platelet aggregation, protection of endothelial cells from damage by free radicals, prevention of calcium ion entry into ischemic cells, decreasing the release of acetylcholine at motor end plates within the neuromuscular junction, and as a competitive antagonist to the glutamate $\mathrm{N}$-methyl-D-aspartate receptor (which is epileptogenic). Anticonvulsant therapy is also used for prevention of seizures in women with mild preeclampsia, but its role in this setting is controversial. The magnesium sulfate dose is $4 \mathrm{~g}$ intravenously as a loading dose then $1 \mathrm{~g} / \mathrm{h}$, or $5 \mathrm{~g}$ intramuscularly into each buttock followed by $5 \mathrm{~g}$ intramuscularly every four hours. Hypotension is the major concern from regional anesthesia since preeclamptic women have depleted intravascular volumes.

Outcome - The major adverse outcomes associated with preeclampsia are related to maternal central nervous system, hepatic, and renal dysfunction (eg, cerebral hemorrhage, hepatic rupture, renal failure), bleeding related to thrombocytopenia, preterm delivery, fetal growth restriction, abruptio placentae, and perinatal death. Factors that influence outcome include gestational age at onset and delivery, severity of disease, and whether there are coexisting conditions present, such as multiple gestation, diabetes mellitus, renal disease, thrombophilia, or preexisting hypertension. There is approximately one maternal death due to preeclampsia-eclampsia per 100,000 live births, with a case-fatality rate of 6.4 deaths per 10,000 cases in US. In mild preeclampsia neonatal outcomes are generally good and comparable to those of normotensive women. Risk of recurrence in subsequent pregnancy depends upon severity of disease, gestational age at onset and gestational age at delivery. 
Postpartum course -Vital signs are monitored every two hours while the patient remains on magnesium sulphate and laboratory tests are repeated until two consecutive sets of data are normal. Hypertension and proteinuria due to preeclampsia resolve postpartum, often within a few days, but sometimes take few weeks or rarely a year or more. Antihypertensive medications can be discontinued when blood pressure returns to normal levels. Elevated blood pressures that remain 12 weeks postpartum are unlikely to be related to preeclampsia and may require long-term treatment. Vasoconstriction and endothelial dysfunction resolve over a few days.

Postpartum preeclampsia Pre eclampsia / eclampsia occurring more than two days and less than six weeks after delivery. It is important to exclude other diagnoses, especially when symptoms suggest the possibility of central nervous system pathology. Late postpartum eclampsia is defined as eclamptic seizures developing greater than 48 hours, but less than four weeks postpartum). Controversial prevention strategies include calcium supplementation, fish oils, sodium restriction, low-dose aspirin and antioxidants.

\subsubsection{Hemolysis, elevated liver enzymes, low platelets -HELLP syndrome}

HELLP syndrome is a variant of severe preeclampsia, first described by Dr. Louis Weinstein in 1982. About 15 to 20 percent of affected patients do not have antecedent hypertension or proteinuria, leading some experts to believe that HELLP is a separate disorder from preeclampsia [Sibai BM1986, Reubinoff BE, 1991]. Both severe preeclampsia and HELLP syndrome may be associated with other hepatic manifestations, including infarction, hemorrhage, and rupture. HELLP develops in approximately 1 to 2 per 1000 pregnancies overall and in 10 to 20 percent of women with severe preeclampsia/ eclampsia. The majority of cases are diagnosed between 28 and 36 weeks of gestation with 70 percent occurring prior to delivery [Sibai BM, Ramadan MK 1993]. Of these patients, approximately 80 percent are diagnosed prior to 37 weeks of gestation and fewer than 3 percent develop the disease between 17 and 20 weeks of gestation. The disease presents postpartum in 30 percent, usually within 48 hours of delivery, but occasionally as long as seven days after birth. Only 20 percent of postpartum patients with HELLP have evidence of preeclampsia antepartum.

\section{Diagnosis and classification of HELLP syndrome}

There is no consensus regarding the degree of laboratory abnormality diagnostic of HELLP syndrome. Some studies use AST (and/or ALT) and LDH values above the upper limit of normal, while others require elevations of at least two standard deviations above the mean. Due to differences in assays used to measure these enzymes, an elevated value in one hospital may be near normal in another.HELLP syndrome and severe preeclampsia are probably part of a disease spectrum. A precise definition of HELLP is necessary for research purposes and for predicting maternal complications. We require the presence of all of the following criteria to diagnose HELLP.

Microangiopathic hemolytic anemia with characteristic schistocytes (also called helmet cells) on blood smear. Other signs suggestive of hemolysis include an elevated LDH or indirect bilirubin and a low serum haptoglobin concentration $(\leq 25 \mathrm{mg} / \mathrm{dL})$.

Platelet count $\leq 100,000$ cells/microL . HELLP syndrome accounts for $21 \%$ of maternal thrombocytopenia in pregnancy.

Serum $\mathrm{LDH} \geq 600 \mathrm{IU} / \mathrm{L}$ or total bilirubin $\geq 1.2 \mathrm{mg} / \mathrm{dL}$ 
Serum AST $\geq 70$ IU/L. Some investigators obtain ALT levels instead of, or in addition to, AST levels. An advantage of the AST is that it is a single test that reflects both hepatocellular necrosis and red cell hemolysis.

Women who do not meet all of the above laboratory abnormalities are considered to have partial HELLP syndrome. These patients may progress to complete expression of HELLP syndrome.

Approximately $50 \%$ of patients have complete HELLP (all components present), and 50\% have incomplete HELLP (at least 1 components present: EL, HEL, ELLP, LP). Some physicians subclassify HELLP based on the severity of thrombocytopenia, as follows: Class 1 - Platelet count $<50,000 / \mu L$, Class 2 - Platelet count 50,000-100,000/ $\mu L$, Class 3 Platelet count $100,000-150,000 / \mu \mathrm{L}$

\section{Clinical manifestations}

Clinical manifestations are nonspecific (nausea, vomiting, headache in 50\%, epigastric or right upper quadrant pain in 50\%).Early HELLP syndrome is often misdiagnosed as heartburn, hence a high index of suspicion for HELLP syndrome is important. All patients with HELLP syndrome do not meet the strict criteria for preeclampsia. Hypertension (blood pressure $\geq 140 / 90$ ) and proteinuria are present in approximately $85 \%, 15 \%$ have diastolic blood pressure (BP) $>90 \mathrm{~mm} \mathrm{Hg}$ but either or both may be absent in women with severe HELLP syndrome (Sibai, 2004). Some patients are asymptomatic, major complications can occur despite normal blood pressure and proteinuria. These include DIC, abruptio placentae, acute renal failure, pulmonary edema, subcapsular liver hematoma, retinal detachment or stroke. Jaundice and ascites may also be present. Bleeding may occur due to thrombocytopenia. Thrombocytopenia is usually moderate, with counts rarely $<20,000 / \mu \mathrm{L}$. Major hemorrhage is uncommon. Maternal thrombocytopenia reaches a nadir at $24-48$ hours postpartum. The maternal mortality rate with HELLP is $1 \%$. The perinatal mortality rate is $11 \%$. Fetal growth restriction is common. Neonates may be at increased risk for thrombocytopenia.

\section{Treatment}

Management is like preecclapsia .Delivery is the ultimate cure and should be planned immediately if $>34$ weeks or can be delayed for $24-48$ hours if $<34$ weeks' gestation to administer corticosteroids if the patient is asymptomatic and the fetal testing is normal. Magnesium sulfate $\left(\mathrm{MgSO}_{4}\right)$ should be administered intrapartum and postpartum, regardless of blood pressure levels, to prevent seizures (eclampsia). Approximately 6-10 units of platelets is administered at the time of skin incision, and an additional 6 units are administered if oozing is noted during the surgery. Also platelet transfusion is indicated if the platelet count is less than 20,000 cells/uL. If cesarean delivery is planned platelet transfusion, is recommended to achieve platelet count greater than 50,000 cells/uL. Thrombocytopenia and elevated liver function tests worsen postpartum but should start normalizing by the third postpartum day. The use of steroids for HELLP is controversial.

Management of hepatic complications - Marked elevations in serum aminotransferases are not typical of uncomplicated HELLP; when they occur, the possibility of hepatic infarction, subcapsular hematoma, or viral hepatitis should be considered. Marked elevation in serum aminotransferases (usually 1000 to $2000 \mathrm{IU} / \mathrm{L}$ or higher) associated with right upper quadrant pain and fever is characteristic of hepatic infarction; this diagnosis can be confirmed by hepatic imaging. These infarcts resolve post partum. These 
patients may have an underlying procoagulant state, such as the antiphospholipid syndrome. HELLP may be complicated by hepatic rupture with development of a hematoma beneath Glisson's capsule. Histology of the liver adjacent to the rupture shows periportal hemorrhage and fibrin deposition, along with a neutrophilic infiltrate( hepatic preeclampsia). The hematoma may remain enclosed, or rupture, with hemoperitoneum . A hepatic hematoma rarely occurs in the absence of preeclampsia or HELLP .It is characterized by abdominal pain and many have severe thrombocytopenia, shoulder pain, nausea, and vomiting. If hepatic rupture occurs, swelling of the abdomen from hemoperitoneum and shock is seen. The aminotransferases are elevated, and values of 4000 to $5000 \mathrm{IU} / \mathrm{L}$ can occasionally be seen. Imaging using CT or MR is more sensitive than ultrasonography for these lesions. The management of a contained hematoma is to support the patient with volume replacement and blood transfusion, as needed, with consideration of percutaneous embolization of the hepatic arteries. It takes months for the hematoma to resolve completely. Surgical intervention is indicated if there is hemodynamic instability, persistent bleeding, increasing pain, or continued expansion of the hematoma. Operative management includes packing, drainage, hepatic artery ligation, and/or resection of affected areas of the liver. For patients with intractable hemorrhage, administration of recombinant factor VIIa and liver transplantation are recommended. Patients who survive have no hepatic sequelae.

Postpartum couse - Decreasing platelet counts continue until 24 to 48 hours after delivery, while serum LDH concentration usually peaks 24 to 48 hours postpartum. An upward trend in platelet count and a downward trend in LDH concentration should be seen by the fourth postpartum day in the absence of complications. Recovery can be delayed in women with DIC, platelet count less than 20,000 cells/uL, renal dysfunction or ascites.

Outcome is generally good; however, serious complications are relatively common. In a series of 437 women with HELLP syndrome at a tertiary care facility, the following complications were observed DIC - $21 \%$, Abruptio placentae - $16 \%$, Acute renal failure $-8 \%$, Pulmonary edema $-6 \%$, Subcapsular liver hematoma $-1 \%$, Retinal detachment $-1 \%$. In addition, $55 \%$ of the patients required blood or blood products, and $2 \%$ required laparotomies for intraabdominal bleeding. $1 \%$ died. Other complications include: adult respiratory distress syndrome, sepsis, and stroke. Wound complications secondary to bleeding and hematomas are common in women with thrombocytopenia. Fetal complications include prematurity $(70 \%)$, intrauterine growth restriction and abruptio placenta, and depend largely upon the severity of the disease and the gestational stage. The overall perinatal mortality is 7 to $20 \%$. However, surviving babies do not have an increased risk of liver disease or thrombocytopenia. Although most liver function tests return to normal postpartum, rarely high total bilirubin may be seen in $20 \%$ even till 3 years post partum. HELLP syndrome with or without renal failure does not affect longterm renal function. The rate of recurrence is $2-27 \%$. Recurrent hepatic rupture in a subsequent pregnancy have been reported, suggesting there may be a genetic predisposition to this condition. Women with a history of HELLP syndrome are at high risk for developing preeclampsia in a subsequent pregnancy. The incidence of preeclampsia varies from 20-50 percent in normotensive women to 75 percent in those with underlying hypertension. There is no evidence that any therapy prevents recurrent HELLP syndrome. 


\subsubsection{Microangiopathies}

Microangiopahy is seen in pre eclampsia. Two other important conditions where microangiopathy in pregnancy is seen are Thrombotic thrombocytopenic purpura (TTP) and hemolytic uremic syndrome (HUS) which are characterized by thrombocytopenia, hemolytic anemia, and multiorgan failure. Incidence is 1 in 25,000 births. TTP is known for central nervous system involvement, while HUS predominantly affects the kidneys. Significant overlap exists in the clinical manifestations of TTP and HUS.

Pathogenesis of microangiopathies -Normally the endothelium is smooth and platelets do not adhere to the endothelium. In microangiopathies the endothelium is damaged and there is formation of thrombi. Complement activation plays an important role in endothelial damage.

Role of complement-Complement is a system of serum proteins.The complement cascade is activated through three pathways (i.e., classical, lectin, and alternative pathways) that converge in the generation of $\mathrm{C} 3$ convertase. Because the alternative pathway is initiated spontaneously, the alternative $\mathrm{C} 3$ convertase $(\mathrm{C} 3 \mathrm{bBb})$ is tightly regulated by plasma and membrane-bound factors, mainly factor $\mathrm{H}(\mathrm{FH})$, factor $\mathrm{I}(\mathrm{FI})$, membrane cofactor protein $(\mathrm{MCP})$, and decay-accelerating factor (DAF).Complement activation leads to endothelial damage.

Role of platelets-Platelets are nonnucleated cells derived from megakaryocytes in the bone marrow and normally live in the peripheral circulation for as long as 10 days. Platelets play a critical initiating role in the hemostatic system. Primary hemostasis begins when platelets adhere to the site of endothelial disruption, leading to platelet clumping. This is followed by platelet activation, characterized by release of granules containing von Willebrand factor, adenosine 5'-diphosphate (ADP), and serotonin. This attracts other platelets into the platelet plug, which stops the bleeding. Simultaneously, the synthesis of thromboxane $A_{2}$ and release of serotonin leads to vasoconstriction to reduce blood loss at the site of vascular injury. The secondary hemostatic phase begins when the coagulation pathway is activated on the surface of the activated platelets to form a secondary thrombus with fibrin. Abnormal intravascular platelet aggregation leads to microthrombi formation, which results in thrombocytopenia, intravascular hemolysis from the breakage of red blood cells through partially occluded vessels, and end organ ischemia.

Genesis of thrombocytopenia-The platelet count in nonpregnant women is 150,000 $400,000 / \mu \mathrm{L}$. Average platelet count in pregnancy is decreased $(213,000 / \mu \mathrm{L}$ vs $250,000 / \mu \mathrm{L})$. Low platelet count is due to hemodilution, increased platelet consumption, and increased platelet aggregation driven by increased levels of thomboxane $\mathrm{A}_{2}$. Thrombocytopenia can be defined as platelet count less than $150,000 / \mathrm{uL}$ or platelet count below the 25 th percentile for pregnant patients $(116,000 / \mu \mathrm{L})$. In pregnancy mild,moderate and severe thrombocytopenia are defined as platelet count of $100,000-150,000 / \mu \mathrm{L} ; 50,000-100,000 / \mu \mathrm{L}$ and $<50,000 / \mu \mathrm{L}$ respectively.

Development of TMA-An acquired or constitutional deficiency in ADAMTS13, a von Willebrand factor (vWF)-processing enzyme, is an important cause of of a peculiar type of TMA. ADAMTS13 levels fall during the last two trimesters of pregnancy, hence TTP is more common at this time. (Fadi Fakhouri, 2010).

Development of HUS-The pathogenesis of pregnancy-associated atypical hemolytic uremic syndrome (P-aHUS) remains obscure and viral illness, retained placental fragments, drugs eg oxytocics, ergot and oral contraceptives have been implicated. The severe deficiency in the von Willebrand factor cleaving protease (ADAMTS13) seen in TTP is rarely present in 
patients with HUS. P-aHUS may be related to alternative C3 convertase dysregulation. A lack of control of the alternative C3 convertase leads to complement-induced lesions of the endothelial cells. Acquired (anti-FH antibodies) or constitutional (inactivating mutations in $\mathrm{FH}, \mathrm{FI}$, or $\mathrm{MCP}$ coding genes or activating mutations in factor $\mathrm{B}$ and $\mathrm{C} 3$ coding genes) with dysregulation of the alternative $\mathrm{C} 3$ convertase has been established as a risk factor for the occurrence of aHUS. It is assumed that excessive activation of the alternative $\mathrm{C} 3$ convertase leads to complement activation. Pregnancy may trigger the onset or subsequent relapses. Mutations in the SCR19-20 domains of factor $\mathrm{H}$ are less frequent in P-aHUS patients compared with non-pregnancy-related aHUS.

\subsubsection{Hemolytic uremic syndrome(HUS)/Idiopathic post partum failure}

It occurs in primipara and is characterized by renal failure, anemia and hypertension. The risk for P-aHUS is highest during a second pregnancy. Onset is within hours to days post partum. Symptoms can begin before delivery, but the onset in most cases is delayed for 48 hours or more after delivery (mean four weeks). HUS may follow a normal pregnancy or be preceded by findings indistinguishable from preeclampsia. HUS with severe renal failure more frequently presents in the postpartum period. Presenting symptoms are often nonspecific , although majority (60\%) present with bleeding. Hypertension is seen in $75 \%$ of cases. Hemolysis and anemia may be absent at presentation in $50 \%$ of cases. DIC is rare.

Labs - Obligate findings include hemolytic anemia (hematocrit $<30 \%$ with schistocytes on peripheral smear) and thrombocytopenia under $100,000 / \mu \mathrm{L}(50 \%$ of patients will have counts $<20,000 / \mu \mathrm{L}$ ). LDHis usually $>600 \mathrm{U} /$ liter. Hemolytic anemia is coomb's negative. There may be hypocomplementemia, deficiency in prostaglandins or antithrombin levels. HUS is a clinical diagnosis. Tissue biopsy is not required. In problematic cases when renal biopsy is done it shows mesangiolysis and glomerular simplification.

Outcomes- The outcomes are poor. $80-90 \%$ survive acute episode. There is a high maternal mortality of $18-44 \%$ and fetal loss is seen in $80 \% .62 \%$ reached ESRD by 1 month. Outcomes do not differ between patients with pregnancy-related and non-pregnancy-related aHUS. Pregnancies in female patients with complement abnormalities are complicated by fetal loss and preeclampsia in $4.8 \%$ and $7.7 \%$,respectively. (Fadi Fakhouri,2010) Almost half have residual neurologic or chronic renal failure. Recurrences occur in $50 \%$. Long-term sequelae, such as hypertension and chronic renal failure, are observed in $44 \%$ of patients with HUS. The perinatal mortality rate is as high as $30 \%$.

Treatment includes Plasmapheresis. Plasma infusion alone is less effective. Steroids, antiplatelet therapy, Immunoabsorption, splenectomy, IV gamma globulin therapy have been used in different studies. Renal failure is managed by hemodialysis. Antihypertensives are used for control of blood pressure. Platelet transfusions should be avoided. Heparin and fibrinolytic agents and anti thrombin III concentrates may be used. Dilatation and curettage should be considered when the disease occurs very close to delivery.

\subsubsection{Thrombotic thrombocytopenic purpura}

TTP is characterized by the pentad of microangiopathic hemolytic anemia, thrombocytopenia, renal insufficiency, fever, and neurologic abnormalities. TTP usually occurs antepartum, about $12 \%$ in the first trimester, $56 \%$ in the second trimester, and $33 \%$ in the third trimester/postpartum(Egerman RS, 1996), There may be mild renal failure and severe neurological involvement (headache, altered consciousness, seizures, hemiparesis), and fever. Severe thrombocytopenia and hemolytic anemia may be seen. Examination 
shows petechiae, ecchymoses, and nose and gum bleeding . Rarely hematuria, gastrointestinal bleeding and intracranial bleeding may occur. Bleeding associated with surgery is uncommon unless the platelet counts are lower than $50,000 / \mu \mathrm{L}$. Clinically significant spontaneous bleeding is rare unless counts fall below $10,000 / \mu \mathrm{L}$.

\section{Treatment}

Plasmapheresis is the recommended therapy. Plasmapheresis removes platelet-aggregating substances causing TTP. Treatment is $90 \%$ successful with TTP but is less successful with HUS. Steroids are used along with plasmapheresis. However, steroids are less effective than plasmapheresis ( $25 \%$ response rate).Platelet transfusions should be avoided when possible because they can cause a clinical deterioration. Platelet transfusions should be used only for uncontrolled bleeding or intracranial hemorrhage. Other therapies include immunosuppressive agents (vincristine, azathioprine, cyclosporine)and splenectomy for TTP. Premature termination of pregnancy has been associated with relapse. Delivery should be considered only when no response to other therapies occurs. (George JN, 2010)

\section{Differential diagnosis}

TTP, HUS and preeclampsia need to be differentiated as prognosis and treatment vary. The distinction between HUS-TTP and severe preeclampsia is often difficult. TTP is characterized by severe thrombocytopenia, hemolytic anemia, neurologic abnormalities (headache, altered consciousness, seizures, hemiparesis), and fever. Renal involvement may occur in $80 \%$ of cases of TTP; TTP occurs in $2^{\text {nd }} / 3^{\text {rd }}$ trimester. von Willebrand factorcleaving protease (ADAMTS-13) activity $<5$ percent is seen in 33 to 100 percent of women with TTP.Unlike HELLP the condition may continue or worsen after delivery with TTP. If suspected preeclampsia/HELLP does not improve within 48-72 hours after delivery, consider TTP. TTP like HUS is associated with isolated platelet consumption; thus, although thrombocytopenia is seen, the other findings of DIC are typically absent. A peripheral blood smear can also be useful. The percentage of schistocytes on peripheral smear is higher in TTP ( 2 to 5 percent).

HUS is characterized by thrombocytopenia, hemolytic anemia, acute renal failure with proteinuria, hematuria, or oliguria/anuria). Neurological manifestations are uncommon. The absence of DIC, onset more than two days after delivery, and/or persistent disease for more than one week are the main findings that characterize HUS. Neurologic involvement may be seen in $50 \%$ of cases of HUS. A peripheral blood smear can also be useful. The percentage of schistocytes on peripheral smear is higher in HUS (2 to 5 percent).ADAMTS deficiency is rare in HUS.

History of preceding proteinuria and hypertension favor preeclampsia, preeclampsiaHELLP does not occur before 20 weeks of gestation and most cases are diagnosed in the third trimester. Delivery leads to resolution with preeclampsia, HELLP is associated with thrombocytopenia, and in severe cases, there may be DIC with prolongation of the PT and aPTT, and reductions in the plasma concentrations of factors V and VIII. The percentage of schistocytes on peripheral smear is low $(<1 \%)$. ADAMTS deficiency is not seen in PIH.

In addition, only a few select laboratories are able to perform complementassociated mutational analysis. Studies of larger heterogeneous populations need to be performed before recommending genetic analysis for patients with pregnancy-associated TTP-HUS. It has not been shown that distinguishing TTP from HUS alters treatment of such patients. 


\subsubsection{Antepartum and post partum hemorrhage}

Significant blood loss due to antepartum or postpartum hemorrhage may cause ischemic injury and lead to obstetric acute renal failure.

Antepartum hemorrhage may be due to placenta praevia or concealed hemorrhage due to abruptio placentae which is usually associated with PIH. Treatment consists of intravascular volume repletion and blood transfusions. If the acute renal failure is due to pre renal intravascular volume depletion and is corrected rapidly the patient may recover without dialysis. However if the insult is prolonged it may lead to acute tubular necrosis for which dialysis is needed.

\subsubsection{Puerperal sepsis}

It is an important cause of AKI. According to World Health Organization puerperal sepsis is defined as infection of the genital tract occurring at any time between the rupture of membranes or labor and the 42 day post partum in which 2 or more of the following are present: pelvic pains, fever oral temperature $38,5^{\circ} \mathrm{C}$ or higher on any occasion, abnormal vaginal discharge (example presence of pus), abnormal smell or foul odour of discharge, delayed uterine involution. The causative organisms are gram positive streptococcus pyogenes, staphylococcus aureus, coliforms,Chlamydia and Clostridium tetani. (Momoh ,2010)Causes are prolonged rupture of membranes, obstructed labour, frequent vaginal examinations, anemia, caesarean section etc. .The source of infection may be from the retained products of conception where there may be a history of foul smelling lochia and the retained products can be demonstrated on imaging. High vaginal swab culture may help to identify the causative organism. Other sources may be urinary tract infection or mastitis. Hence a detailed physical examination including the breast examination should be undertaken. Treatment consists of appropriate antibiotics according to the culture sensitivity reports. Surgery to remove the retained products or abscess drainage even hysterectomy in extreme cases may be needed.

\subsubsection{Urinary Tract Infections (UTI)}

Another important cause of renal failure in pregnancy is UTI. UTIs are the most common renal disease occurring during pregnancy and range from asymptomatic bacteriuria to pyelonephritis. UTIs have been associated with SGA babies, premature labor, IUD, anemia and hypertension in mother. In some cases upper UTI may be associated with renal failure. Pregnant females are at risk for development of UTIs (2-10\%), because of anatomic and physiologic changes that occur in normal pregnancy.UTI may be classified as:

\subsubsection{Asymptomatic bacteriuria}

Bacteriuria occurs in 2 to $7 \%$ of pregnancies, particularly in multiparous women, a similar prevalence as seen in nonpregnant women. A clean-voided specimen containing more than 1 lakh organisms per milliliter suggests infection. Bacteriuria often develops in the first month of pregnancy and is frequently associated with a reduction in concentrating ability. $30 \%$ of patients develop pyelonephritis if asymptomatic bacteriuria is left untreated. Universal screening is therefore recommended in all pregnant females. Screening for asymptomatic bacteriuria should be performed at 12 to 16 weeks gestation (or the first prenatal visit, if that occurs later). Rescreening is generally not performed in low risk women, but can be considered in women at high risk for infection (eg, presence of urinary tract anomalies, hemoglobin S, or preterm labor). Dipstick has a sensitivity of $<50 \%$ and 
routine microscopy and culture are needed. Urinalysis with culture should be performed on a monthly basis after resolution. Treatment with a course of oral antibiotics (Nitrofurantoin $100 \mathrm{mg}$ orally every 12 hours for five to seven days, Ampicillin ,Amoxicillin (500 mg orally every 12 hours for three to seven days), Amoxicillinclavulanate ( $500 \mathrm{mg}$ orally every 12 hours for three to seven days),Cephalexin ( $500 \mathrm{mg}$ orally every 12 hours for three to seven days) and Fosfomycin ( $3 \mathrm{~g}$ orally as a single dose)and sulphonamides are some of the safe regimes. Treatment reduces incidence of pyelonephritis to $3 \%$. Fluoroquinolones should be avoided in pregnancy. The safest course is to avoid using nitrofurantoin in the first trimester if another antibiotic that is safe and effective is available. Sulfonamides should be avoided in the last days before delivery because they can increase the level of unbound bilirubin in the neonate. Trimethoprim (FDA category C) is generally avoided in the first trimester because it is a folic acid antagonist, has caused abnormal embryo development in experimental animals, and some case control studies have reported a possible association with a variety of birth defects. Ceftrioxone should be avoided the day before parturition because it displaces bilirubin and may cause kernicterus. Recurrence occurs in 35\%. If bacteriuria is persistent, suppressive therapy (Nitrofurantoin $50-100 \mathrm{mg}$ at night, cephalexin $250-500 \mathrm{mg}$ or amoxycillin) is indicated.

\subsubsection{Cystitis}

Cystitis (3\%) is associated with dysuria, urgency and frequency, without systemic signs. It usually occurs in $2^{\text {nd }}$ trimester and is common even in patients with negative urine cultures. Though cystitis recurs in $17 \%$ it does not progress to acute pyelonephritis. It should be aggressively treated with oral antibiotic regimens. The symptoms of cystitis and pyuria accompanied by a "sterile" urine culture finding may be due to Chlamydia trachomatis urethritis. There may be mucopurulent cervicitis, hence erythromycin therapy is effective.

\subsubsection{Pyelonephritis-(3\%)}

Majority (70\%) patients with PN have asymptomatic bacteriuria. 50\% cases occur in 2 nd trimester. Onset is abrupt with fever, chills, flank pain, anorexia, nausea, vomiting and costovertebral tenderness. The etiologic organisms include Escherichia coli, Klebsiella, Enterobacter and Proteus . 10\% are due to Gram+ organsisms. 15\% patients have concurrent bacteremia. Other complications include hemolysis, sepsis, adult respiratory distress syndrome, hepatic dysfunction and death. Pyelonephritis requires hospitalization and intravenous antibiotics and fluids till fever resolves. Effective regimens include ampicillin plus gentamicin or a third-generation cephalosporin followed by oral administration of antibiotics for 14 days .This leads to complete resolution of infection in $70 \%$. Antibiotics can be further adjusted according to culture sensitivity results. In infections recurring in 2 weeks, 2-3 wks of treatment and suppressive therapy throughout the pregnancy should be given. Post coital cephalexin 250mg or NFT $50 \mathrm{mg}$ may be preventive. Perinephric or cortical abscesses may be seen. Although renal function is well maintained during acute pyelonephritis, some pregnant women develop acute renal failure. Renal biopsy may reveal focal microabscesses and recovery after antimicrobial therapy may be incomplete due to irreversible injury.

\subsubsection{Acute Fatty Liver of Pregnancy (AFLP)}

It is a rare complication of pregnancy. AFLP is unique to pregnancy. There is no predilection for any geographical area or race. It occurs more commonly in nullipara and primipara than 
multipara. It was first described in 1940 by Sheehan as an "acute yellow atrophy of the liver". The incidence of AFLP is 1 case per 20,000 births in the UK. Incidence of AFLP from non-UK studies based on hospital case series estimate 1 in 4000 deliveries to 1 in 16000 births. AFLP is associated with AKI in $60 \%$. AFLP occurs in the third trimester or the immediate period after delivery however it can be seen as early as 26 weeks of gestation. (Buytaert,1996) It is due to mutation in enzyme for long chain 3 hydroxy acyl CoA dehydrogenase leading to disordered metabolism of fatty acids by mitochondria in the mother. The most common mutation found in acute fatty liver of pregnancy is the E474Q missense mutation (IJlst L,1997) This gene mutation is recessive; therefore, in non pregnant state, women have normal fatty acid oxidation. However, if the fetus is homozygous for this mutation, it will be unable to oxidize fatty acids. These acids are passed to the mother, who, because of diminished enzyme function, cannot metabolize the additional fatty acids. This results in hepatic strain leading to the development of AFLP, which can be relieved by delivery of the infant. The accumulation of long-chain 3-hydroxyacyl metabolites produced by the fetus or placenta is toxic to the liver and may be the cause of the liver disease. It is characterized by jaundice, hypoalbuminemia, mild renal failure, DIC, PIH, hypoglycemia, pancreatitis and encephalopathy. Laboratory findings may be consistent with disseminated intravascular coagulation (DIC), specifically, prolongation of prothrombin time, low fibrinogen, and low antithrombin levels. This results in a clinical picture similar to DIC; however, in AFLP, the values are abnormal, not due to consumption of the clotting factors but rather to decreased production by the damaged liver. Bilirubin levels are elevated. This elevation is primarily the conjugated form, with levels exceeding $5 \mathrm{mg} / \mathrm{dL}$. This can result in jaundice, which is rarely seen in patients with other forms of pregnancy-related hepatic injury, including preeclampsia. The diagnosis should be suspected in a woman with preeclampsia who has hypoglycemia, hypofibrinogenemia, and a prolonged PTT in the absence of abruptio placentae, high bilirubin, normal/ high transaminases, microvesicular steatosis and rarely liver necrosis. Criteria for diagnosis of AFLP include six or more of the following features in the absence of another explanation: Vomiting, abdominal pain, polydipsia / polyuria, encephalopathy, elevated bilirubin, hypoglycemia, elevated urate, leucocytosis $(>11 \times 109 / 1)$, ascites or bright liver on ultrasound scan, elevated transaminases (aspartate aminotransferase or alanine aminotransferase $>42 \mathrm{IU} / 1$ ), elevated ammonia, renal impairment, coagulopathy (prothrombin time $>14 \mathrm{~s}$ or activated partial thromboplastin time $>34 \mathrm{~s}$ ), microvesicular steatosis on liver biopsy. (Ch'ng,2002). The liver biopsy is diagnostic but is not always feasible especially in patients with severe coagulopathy (Bacq, 2006) and it seldom influences acute management. Ultrasound and computed tomography have been used but false negative results arecommon. Renal biopsy shows Acute tubular necrosis (ATN). Delivery of the fetus, regardless of gestational age, is the only treatment for acute fatty liver of pregnancy (AFLP) once the diagnosis has been made. Treatment includes intravenous fluids, cryoprecipitate, Fresh frozen Plasma and glucose. It reverses with delivery. $\mathrm{C}$ section is preferred over vaginal delivery. Liver transplant is the treatment of choice in patients with liver necrosis. There is $20-25 \%$ maternal and fetal mortality and the syndrome can recur in subsequent pregnancies with a calculated genetic risk of $25 \%$.The other causes of jaundice during pregnancy include cholestasis, cholelithiasis, viral hepatitis and pre-eclampsia with or without HELLP syndrome. Intrahepatic cholestasis of pregnancy may present during the third trimester but itching is the characteristic symptom and serum bilirubin concentration is rarely higher than $6 \mathrm{mg} / \mathrm{dl}$. Cholelithiasis may occur at any time during pregnancy and is accompanied by pain in the right upper quadrant, and fever, and 
USG is usually diagnostic. Acute viral hepatitis in pregnancy presents as a systemic illness withfever, nausea, vomiting, fatigue, and jaundice, however, aminotransferase concentrations are markedly elevated $(>500 \mathrm{U} /$ liter). The manifestations of pre-eclampsia are usually observed in the second half of pregnancy, whereas the symptoms of HELLP syndrome and AFLP frequently appear in the third trimester. The incidence of HELLP syndrome is much higher $(1: 5,000)$. Morbidity of the infant includes increased risk of cardiomyopathy, neuropathy, myopathy, nonketotic hypoglycemia, hepatic failure, and death associated with fatty acid oxidation defects in newborns.

\subsubsection{Urinary tract obstruction}

Functional hydronephrosis rarely cause renal failure. The diagnosis can be established in some cases by the normalization of renal function in the lateral recumbent position and its recurrence when supine. In some cases, either insertion of a ureteral catheter or delivery of the fetus is required. Rarely, acute urinary tract obstruction in pregnancy is induced by a kidney stone.

\subsubsection{Antiphospholipid Syndrome (APS)}

It refers to a syndrome characterized by arterial or venous thrombosis or specific pregnancy complications in women with laboratory evidence of antibodies to proteins bound to anionic phospholipids. International consensus conferences have proposed and revised classification criteria for definite APS ( Sapporo criteria)(Miyakis 2006). Definite APS is considered present if at least one of the following clinical criteria and at least one of the following laboratory criteria are satisfied.

1. Clinical - (a)Thrombosis - Unequivocal imaging or histologic evidence of thrombosis in any tissue or organ, OR (b) Pregnancy morbidity - Otherwise unexplained death at $\geq 10$ weeks gestation of a morphologically normal fetus, OR One or more premature births before 34 weeks of gestation because of eclampsia, preeclampsia, or placental insufficiency, OR Three or more embryonic ( $<10$ week gestation) pregnancy losses unexplained by maternal or paternal chromosomal abnormalities or maternal anatomic or hormonal causes.

2. Laboratory - The presence of antiphospholipid antibodies (aPL), on two or more occasions at least 12 weeks apart and no more than five years prior to clinical manifestations, as demonstrated by one or more of the following: (a)IgG and/or IgM aCL in moderate or high titer ( $>40$ units GPL or MPL or $>99$ th percentile for the testing laboratory),(b)Antibodies to ß2-glycoprotein-I (B2-GP-I) of IgG or IgM isotype at a titer $>99$ th percentile for the testing laboratory when tested according to recommended procedures.(c)Lupus anticoagulant (LA) activity detected according to published guidelines

Clinical manifestations include deep vein thrombosis, thrombocytopenia, livedo reticularis, stroke, superficial thrombophlebitis, pulmonary embolism, fetal loss, transient ischemic attack, and hemolytic anemia. In rare patients, APS results in multiorgan failure because of multiple blood vessel occlusions, a condition referred to as "catastrophic antiphospholipid syndrome" In patients with preeclampsia or the HELLP syndrome, catastrophic APS must be considered, in patients with histories of thrombosis or spontaneous abortions. Thrombotic renal disease occurs in a minority of patients with APS. Glomerular capillaries and other renal vessels, both arteries and veins of all sizes, can be affected. The disease may 
be silent or produce acute or chronic renal failure with proteinuria. APLS may cause glomerular lesions like membranous nephropathy, minimal change disease or proliferative glomerulonephritis.

\section{Laboratory diagnosis}

A history of a biologic false positive serologic test for syphilis (BFPTS) may be a clue to the presence of any type of aPL: aCL, B2-GP-I antibodies, or an LA. However, because of the nonspecific nature of the BFPTS, the presence of one or more aPL should be confirmed with one of the tests (A)The presence of aPL may be demonstrated directly by: ELISA testing in the case of aCL and antibodies to B2-GP-I and (B) Clotting assay that demonstrates effects of an aPL on the phospholipid-dependent factors in the coagulation cascade (LA test).The lupus anticoagulant phenomenon refers to the ability of aPL to cause prolongation of in vitro clotting assays such as the activated partial thromboplastin time (aPTT), the dilute Russell viper venom time (dRVVT), the kaolin clotting time or, the prothrombin time. This prolongation is not reversed when the patient's plasma is diluted 1:1 with normal plateletfree plasma.

Treatment includes heparin, oral anticoagulants ie warfarin, antiplatelet drugs eg aspirin and clopidogrel and hydroxyl chloroquin.

\subsubsection{Miscellaneous causes}

The other miscellaneous causes of acute renal failure in pregnancy include Acute gastroenteritis, infections, drugs and other causes similar to the varied causes of AKI in non pregnant states.

\subsection{Pathology}

Pathology of pregnancy related AKI varies with etiology.

\subsubsection{Glomerular endotheliosis}

It is the typical finding in PIH. Glomeruli look enlarged and the endothelial cells are swollen. This lesion was first described by Spargo. It resolves by the end of post partum period.

\subsubsection{Acute tubular necrosis}

May be seen in cases of ischemic or toxic insult.

\subsubsection{Acute interstitial nephritis}

May be commonly due to infection or drug.

\subsubsection{Cortical necrosis}

It is one of the dreaded histopathological lesions . $50 \%$ of all cortical necrosis is pregnancy related. Causes include Abruptio (esp if concealed hemorrhage), septic abortion, placenta previa, prolonged IUD, or amniotic fluid embolism. It is commoner in post partum than antepartum AKI. Renal cortical necrosis may be patchy or total. The triad of anuria, gross hematuria, and flank pain seen in cortical necrosis is unusual in the other causes of renal failure in pregnancy. The diagnosis can be established by ultrasonography which shows a subcapsular hypoechoic band . Renal calcifications on plain film of the abdomen suggest 
cortical necrosis (6 weeks). CT scanning demonstrates hypoechoic or hypodense areas in renal cortex or may demonstrate cortical tram track calcification by 3 weeks. Angiogram shows abrupt cut off of vascularity which is called pruning. No specific therapy is effective. Many patients develop chronic kidney disease. In our study from Osmania General Hospital, Hyderabad, India over 7 years there were total of 105 patients with Renal cortical necrosis (RCN). The mean age was $28.125 \pm 12.40$ years. Forty one cases $(39.04 \%)$ resulted from obstetric complications. The most common histology type of RCN was patchy cortical necrosis in 65 patients (62\%). All patients required dialysis and the mean duration of dialysis was $3 \pm 1$ weeks. Thirty three $(31.42 \%)$ patients progressed to end stage kidney disease while 3 underwent renal transplantation. 10(9.5\%) patients succumbed to the acute kidney injury (AKI).(Manisha Sahay)

\subsubsection{Vascular changes}

May be seen in HUS with glomerular and arteriolar fibrinoid necrosis and thrombi. Glomeruli show mesangiolysis and glomerular simplification.

\subsection{Dialysis in pregnancy}

Early dialysis is necessary in pregnant women with renal failure and should be considered when the serum creatinine reaches $3.5 \mathrm{mg} / \mathrm{dL}$ or the glomerular filtration rate (GFR) is less than $20 \mathrm{~mL} / \mathrm{min}$ or blood urea nitrogen is more than $100 \mathrm{mg} / \mathrm{dl}$. Longer, more frequent dialysis $(20 \mathrm{~h} / \mathrm{wk})$ is associated with the best fetal outcome. Hemodialysis may therefore be necessary at least 5 days per week. Careful avoidance of hypotension is important. As pregnancy is a procoagulant state the dose of heparin needs to be increased. Peritoneal dialysis with smaller volumes and frequent exchanges is another option. Peritoneal dialysis may be difficult in the third trimester due to increased uterine size. Premature labor and fetal size that is small for the gestational age are typical in women who deliver on dialysis. Nutritional support that allows weight gains of 0.3 to $0.5 \mathrm{~kg} / \mathrm{wk}$ should be maintained in the second and third trimesters. Although the spontaneous abortion rate is approximately $50 \%$ for pregnant women who require dialysis, the fetal survival rate for pregnancies that continue is as high as $71 \%$.

\section{References}

ACOG practice bulletin. Diagnosis and management of preeclampsia and eclampsia. Number 33, January 2002.ACOG Committee on Practice Bulletins Obstetrics,Obstet Gynecol. 2002;99(1):159.

American College of Obstetrics and Gynecology (2004)ACOG (American College of Obstetrics and Gynecology) Practice Bulletin: nausea and vomiting of pregnancy. Obstet Gynecol. ;103(4):803.

August P, Mueller FB, Sealey JE, Edersheim TG. (1995) Role of rennin angiotensin system in blood pressure regulation in pregnancy. Lancet , 345:896-897.

Ananth Karumanchi S ,Franklin H Epstein (2007)Renal complications in pregnancy In Comprehensive Clinical Nephrology: edn $3^{\text {rd }}$ Edited by John Feehally, Jurgen Floege, Richard J. Johnson .Mosby Elsevier .483-504. 
Bacq Y. (2006)The liver in pregnancy. In: Schiff ER, Sorrell MF, Schiff L, Maddrey WC, editors. Schiff's Diseases of the liver. $10^{\text {th }}$ ed. Lippincott Williams and Wilkins (LWW); .p. 1281-1304.

Baylis C: (1987) Glomerular filtration and volume regulation in gravid animal models. Clin Obstet Gynaecol , 1:789.

Baylis,C, John M Davidson: Renal Physiology in normal pregnancy.(2007) In Comprehensive Clinical Nephrology: edn $3^{\text {rd }}$ Edited by John Feehally, Jurgen Floege, Richard J. Johnson .Mosby Elsevier,475-481.

Beaufils MB. Pregnancy. In: Davidson AM, Cameron JS, Grunfeld JP, et al., editors. (2005)Clinical nephrology. 3rd ed. New York: Oxford University Press; pp. 1704-28.

Buytaert IM; Elewaut GP; Van Kets HE. (1996) Early occurrence of acute fatty liver in pregnancy. Am J Gastroenterol Mar; 91(3):603-4.

Ch'ng CL, Morgan M, Hainsworth I, et al. (2002)Prospective study of liver dysfunction in pregnancy in Southwest Wales. Gut; 51: 876-80.

Chugh KS. (1987) Etiopathogenesis of acute renal failure in the tropics. Ann Natl Acad Med Sci (India);23:88-99.

Egerman RS, Witlin AG, Friedman SA, Sibai BM. (1996) Thrombotic thrombocytopenic purpura and hemolytic uremic syndrome in pregnancy: review of 11 cases. Am J Obstet Gynecol. ;175(4 Pt 1):950-6.

Fadi Fakhouri, Lubka Roumenina,Franc, ois Provot,Marion Salle’ e,Sophie Caillard, Lionel Couzi,Marie Essig, David Ribes, Marie-Agne`s Dragon-Durey, Frank Bridoux,Eric Rondeau, and Veronique Fre' meaux-Bacchi (2010)Pregnancy-Associated Hemolytic Uremic Syndrome Revisited in the Era of Complement Gene Mutations J Am Soc Nephrol 21: 859-867.

George JN (2010) How I treat patients with thrombotic thrombocytopenic purpura: Blood.;116(20):4060.

Hou S. (1998) The kidney in pregnancy. In: Greenberg A. Primer on Kidney Diseases. 2nd ed. Academic Press; :388-394.

IJlst L, Oostheim W, Ruiter JP, Wanders RJ (1997). "Molecular basis of long-chain 3hydroxyacyl-CoA dehydrogenase deficiency: identification of two new mutations". J. Inherit. Metab. Dis. 20 (3): 420-422

Levine, RJ, Lam, C, Qian, C, et al. (2006) Soluble endoglin and other circulating antiangiogenic factors in preeclampsia. N Engl J Med; 355:992.

Lindheimer MD, Katz AI(1991) : The kidney and hypertension in pregnancy.In The Kidney, edn 4. Edited by Brenner BM, Rector FC. Philadelphia:WB Saunders Co; :1551-1595.

Levi M (2009) Disseminated intravascular coagulation (DIC) in pregnancy and the peripartum period. Thromb Res. 2009;123 Suppl 2:S63.

Lucas MJ, Leveno KJ, Cunningham FG (1995) A comparison of magnesium sulfate with phenytoin for the prevention of eclampsia. N Engl J Med. 333(4):201.

Manisha Sahay Cortical necrosis in tropics, accepted for publication, Saudi journal of Kidney disease and transplantation .

Maynard S, Min J, Merchan J, Lim K, Li J, Mondal S, Libermann T, Morgan J, Sellke F, Stillman I, Epstein F, Sukhatme V, Karumanchi S (2003). "Excess placental soluble fms-like tyrosine kinase 1 (sFlt1) may contribute to endothelial dysfunction, hypertension, and proteinuria in preeclampsia". J Clin Invest 111 (5): 649-58. 
M.A. Momoh, O.J. Ezugworie and H.O. Ezeigwe (2010) Causes and Management of Puerperal Sepsis: The Health Personnel View Point Advances in Biological Research 4 (3): 154-158.

Miyakis S, Lockshin MD, Atsumi T, Branch DW, Brey RL, Cervera R, Derksen RH, DE Groot PG, Koike T, Meroni PL, Reber G, Shoenfeld Y, Tincani A, Vlachoyiannopoulos PG, Krilis SA(2006) International consensus statement on an update of the classification criteria for definite antiphospholipid syndrome (APS).J Thromb Haemost. ;4(2):295.

Naqvi R, Akthar F, Ahmad E, Shaikh R, Ahmed Z, Naqvi A, et al. (1996) Acute renal failure of obstetrical origin during 1994 at one centre. Ren Fail. ;18:681-3.

NHBPEP. Report of the National High Blood Pressure Education Program Working Group on High Blood Pressure in Pregnancy. (2000) Am J Obstet Gynecol. ;183(1):S1-S22.

Reubinoff BE, Schenker JG. HELLP syndrome--a syndrome of hemolysis, elevated liver enzymes and low platelet count--complicating preeclampsia-eclampsia. Int J Gynaecol Obstet 1991; 36:95.

Schrier RW, Gottschalk C. Kidney diseases in pregnancy. (1997) In: Diseases of The Kidney. 6th ed. Lippincott Williams \& Wilkins.

Sheehan HL. The pathology of acute yellow atrophy and delayed chloroform poisoning. J Obstet Gynaecol Br Emp. 1940;47:49-62.

Sibai, BM, Villar, MA, Mabie, BC. (1990) Acute renal failure in hypertensive disorders of pregnancy. Pregnancy outcome and remote prognosis in thirty-one consecutive cases. Am J Obstet Gynecol ; 162:777.

Sibai, BM, Ramadan, MK. (1993) Acute renal failure in pregnancies complicated by hemolysis, elevated liver enzymes, and low platelets. Am J Obstet Gynecol ; 168:1682.

Stone JH. (1998) HELLP syndrome: hemolysis, elevated liver enzymes, and low platelets. JAMA 280:559.

Sibai BM, Taslimi MM, el-Nazer A, et al. (1986) Maternal-perinatal outcome associated with the syndrome of hemolysis, elevated liver enzymes, and low platelets in severe preeclampsia-eclampsia. Am J Obstet Gynecol; 155:501.

Sibai BM. (1990) The HELLP syndrome (hemolysis, elevated liver enzymes, and low platelets): much ado about nothing? Am J Obstet Gynecol; 162:311.

Sibai BM, Ramadan MK, Usta I, Salama M, Mercer BM, Friedman SAAm J Obstet Gynecol. 1993Maternal morbidity and mortality in 442 pregnancies with hemolysis, elevated liver enzymes, and low platelets (HELLP syndrome);169(4):1000.

Sibai BM (2004) Diagnosis, controversies, and management of the syndrome of hemolysis, elevated liver enzymes, and low platelet count. Obstet Gynecol.;103 (5 Pt 1):981.

Venkatesha, S; Toporsian M, Lam C, Hanai J, Mammoto T, Kim YM, Bdolah Y, Lim KH, Yuan HT, Libermann TA, Stillman IE, Roberts D, D'Amore PA, Epstein FH, Sellke FW, Romero R, Sukhatme VP, Letarte M, Karumanchi SA. (2006). "Soluble endoglin contributes to the pathogenesis of preeclampsia". Nat Med 12 (6): 642-9.

World Health Organization. 2009. Managing puerperal sepsis. Geneva Switzerland: WHO press.

Zhou, CC, Zhang, Y, Irani, RA, et al. (2008) Angiotensin receptor agonistic autoantibodies induce pre-eclampsia in pregnant mice. Nat Med ; 14:855. 


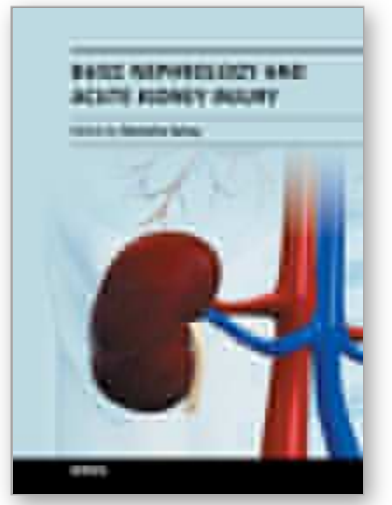

\author{
Basic Nephrology and Acute Kidney Injury \\ Edited by Prof. Manisha Sahay
}

ISBN 978-953-51-0139-0

Hard cover, 226 pages

Publisher InTech

Published online 02, March, 2012

Published in print edition March, 2012

The first section of the book covers the basics of nephrology and second section focuses on acute kidney injury. This easy to reference text examines the physiological and biochemical aspects of renal diseases - all in one convenient resource. Experts in the field discuss topics of increasing concern in nephrology including newer methods of assessing renal function. The field of acute kidney injury in nephrology is a rapidly evolving one with research translating into clinical guidelines and standards. This text brings together experts to provide an authoritative reference for management of AKI in various clinical settings. Pregnancy related AKI is an important entity which has also been discussed in detail. The recent advances in the field of critical care AKI have been incorporated as well and help the reader to update their knowledge.

\title{
How to reference
}

In order to correctly reference this scholarly work, feel free to copy and paste the following:

Manisha Sahay (2012). Acute Kidney Injury in Pregnancy, Basic Nephrology and Acute Kidney Injury, Prof. Manisha Sahay (Ed.), ISBN: 978-953-51-0139-0, InTech, Available from:

http://www.intechopen.com/books/basic-nephrology-and-acute-kidney-injury/acute-kidney-injury-in-pregnancy

\section{INTECH}

open science | open minds

\section{InTech Europe}

University Campus STeP Ri Slavka Krautzeka 83/A 51000 Rijeka, Croatia Phone: +385 (51) 770447

Fax: +385 (51) 686166 www.intechopen.com

\section{InTech China}

Unit 405, Office Block, Hotel Equatorial Shanghai No.65, Yan An Road (West), Shanghai, 200040, China 中国上海市延安西路65号上海国际贵都大饭店办公楼 405 单元 Phone: $+86-21-62489820$

Fax: $+86-21-62489821$ 
(C) 2012 The Author(s). Licensee IntechOpen. This is an open access article distributed under the terms of the Creative Commons Attribution 3.0 License, which permits unrestricted use, distribution, and reproduction in any medium, provided the original work is properly cited. 